\title{
LATE ONSET SEGMENTAL NEUROFIBROMATOSIS \\ IMOSAIC NEUROFIBROMATOSIS TYPE 1]: A CASE REPORT WITH REVIEW OF \\ LITERATURE
}

\author{
Rana $A^{1}$, Joshi $S^{1}$, Pudasaini $S^{2}$
}

${ }^{1}$ Department of Dermatology, ${ }^{2}$ Department of Pathology, Nepal Medical College Teaching Hospital, Attarkhel, Gokarneshwor-8, Kathmandu, Nepal

\begin{abstract}
Segmental neurofibromatosis (SNF) is a rare manifestation of neurofibromatosis. Very few cases have been reported in older population. Here, we present a case of 73 year old male with four years history of multiple, skin coloured, dome shaped, soft nodules situated over left lumbar region. The histopathological evaluation of the excised nodule revealed neurofibroma. Recognition of this condition is important, as there may be associated systemic involvement, malignancies and possibility of transmission to the offspring.
\end{abstract}

\section{KEYWORDS}

Mosaic neurofibromatosis type 1, nodule, segmental neurofibromatosis, Nepal

\section{CORRESPONDING AUTHOR}

Dr. Arnija Rana,

Department of Dermatology

Nepal Medical College Teaching Hospital,

Attarkhel, Gokarneshwor-8, Kathmandu, Nepal.

Email-arnija.ajju@gmail.com 


\section{INTRODUCTION}

Segmental neurofibromatosis (SNF) is a rare manifestation of neurofibromatosis characterized by cutaneous lesions usually limited to one body region, and an absence of systemic complication in the majority of patients. ${ }^{1}$ The estimated prevalence of SNF in the general population is 1 in 36,000-40,000 individuals. ${ }^{2,3}$

In SNF, there is a risk of developing neurofibromatosis type 1 (NF1) in the offspring with systemic involvement like oculocutaneous lesions, bony abnormalities, seizure, and learning difficulties. ${ }^{1,4}$

\section{CASE REPORT}

A 73 years old male, presented in Dermatology Outpatient Department with four years history of skin lesions distributed over the left lower abdomen. The lesions were asymptomatic, however he was curious about the prognosis. He was unmarried and there was no history of similar lesions in his siblings and parents.

On cutaneous examination, multiple soft to firm, scattered, dome shaped skin coloured nodules ranging $3-10 \mathrm{~mm}$ in size were seen in the left lumbar region (Fig. 1). Few of them showed a positive button hole sign. He did not have any ocular, bony or cutaneous features of NF1.

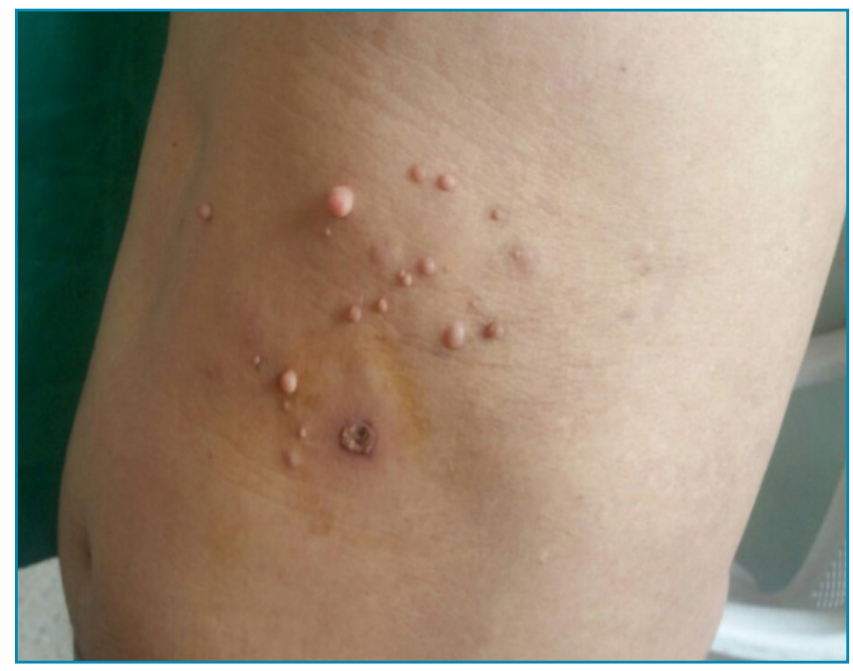

Fig. 1: Multiple skin coloured dome shaped nodules over left lumbar region with an electrofulguration scar in situ.

Electrofulguration of the base of one of the lesion was done. Thus, the sample obtained was sent for histopathological examination. Histopathological examination with Hematoxylin and Eosin (H \& E) stained slide showed a well circumscribed mass composed of a haphazard proliferation of spindle cells in the dermis. These cells were uniform with elongated wavy nuclei showing pointed ends and moderate cytoplasm. The periphery of the tumor showed fibrillary background. There was no evidence of dysplasia/malignancy (Fig. 2).

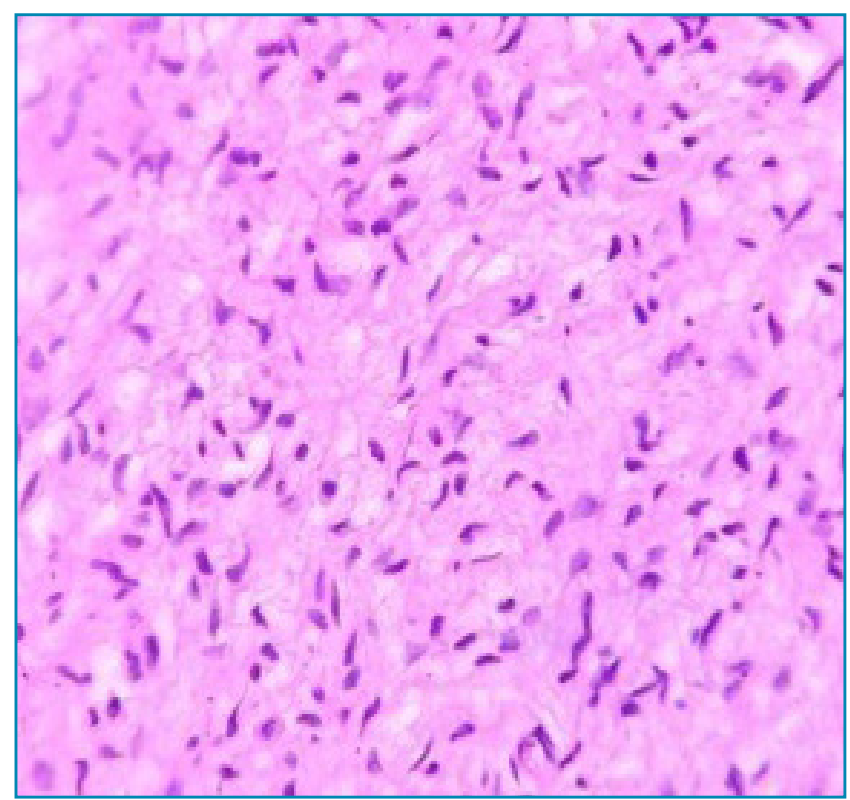

Fig. 2: Uniform spindle cells with elongated wavy nuclei showing pointed ends and moderate cytoplasm in a haphazard background in the dermis.

(H\&E stain, $\mathrm{x} 400$ )

According to clinical and histopathologic features, the diagnosis of SNF was made. Chest X-ray PA view showed patchy radio opacity in the right lung upper zone. An apical chest X-ray was advised, which showed features of calcified focus in right upper lobe, suggestive of an old healed granuloma. Ultrasonography of the abdomen and pelvis showed normal findings.

Surgical removal of the skin lesions was suggested as a treatment modality for aesthetic purpose. A regular medical surveillance was advised.

\section{DISCUSSION}

Riccardi established the diagnostic criteria for SNF: pigmentary abnormalities, which includes café-au lait macules and/or axillary freckles, and/ or neurofibromas in a single unilateral segment of the body, with no crossing of midline, no family history of neurofibromatosis and no systemic involvement. ${ }^{5}$ Roth et $a l^{6}$ further divided SNF into four subtypes: true segmental, localized with deep involvement, hereditary, and bilateral. Segmental neurofibromatosis is also categorized according to the observed clinical manifestations into pigmentary anomalies only, pigmentary anomalies and neurofibromas, neurofibromas only, and plexiform neurofibromas only. ${ }^{2,7}$ The most common presentation of SNF is neurofibroma alone, often in a dermatomal distribution. ${ }^{2,8}$

Genetic testing has proven SNF to be the result of a postzygotic NF1 gene mutations, and the phenotype appropriately called mosaic neurofibromatosis type 1 (MNF1) ${ }^{2,7}$ Based on the distribution of the lesions, the MNF1 can be categorized as localized or generalized. Localized MNF1 denotes clinical 
findings in one segment of the body. In generalized more than one segment of the body is involved, while still in a segmental configuration. ${ }^{2}$

Individuals with MNF1 may have gonadal mosaicism and offsprings with complete NF1. ${ }^{9}$ In the individual case reports of a systematic review on MNF1, majority of cases had neurofibromas only. Also, the cases that presented at the oldest age was the group with neurofibromas only. ${ }^{10}$

The incidence of malignancy in patients with MNF1 is approximated to be $5.3 \% .{ }^{11}$ There are very few reported cases of late onset MNF1 associated with internal malignant tumors. ${ }^{12-14}$ Dang et al $^{12}$ reported a 57 years old male with SNF of the distal arm, who had developed Hodgkin Lymphoma. Another case of SNF in a 66 years old male with meningioma and gastric MALT lymphoma was reported by Bruker et al..$^{13}$ Yalcin et al ${ }^{14}$ reported a 75 years old female with SNF and the concomitant appearance of bronchoalveolar carcinoma of left lower lobe.
An age appropriate and closer screening in adults with SNF is required as there may be systemic associations and increased risk for malignancies than the general population. ${ }^{13}$

This case describes a subtle finding of SNF to which a dermatologist may be exposed. In a suspected SNF patient, a thorough family history with a clinical examination must be undertaken. More specific investigations and workup may be required. In the reported case, no any associated systemic involvement or internal malignancies were found. A long term follow up is recommended for a better knowledge of the clinical course and prognosis of the condition.

There are no specific management guidelines for SNF in the literature. The patient must be informed that SNF is not a generalized form of NF1. Also, such patient should be informed about the small risk of having a child with generalized NF1 in the future.

\section{REFERENCES}

1. Riccardi VM. Neurofibromatosis: clinical heterogeneity. Curr Probl Cancer 1982; 7: 1-34.

2. Ruggieri M, Huson SM. The clinical and diagnostic implications of mosaicism in the neurofibromatoses. Neurol 2001; 56: 1433-43.

3. Ingordo $\mathrm{V}$, D'Andria $\mathrm{G}$, Mendicini $\mathrm{S}$ et al. Segmental neurofibromatosis: is it uncommon or underdiagnosed? Arch Dermatol 1995; 131: 959-60.

4. Boltshauser E, Stocker H, Mächler M. Neurofibromatosis type 1 in a child of a parent with segmental neurofibromatosis (NF-5). Neurofibromatosis 1989; 2: 244-5.

5. Ricchardi VM. Early manifestations of neurofibromatosis: diagnosis and management. Compr Ther 1982; 8: 35.

6. Roth RR, Martines R, James WD. Segmental neurofibromatosis: Arch Dermatol 1987; 123: 91720.

7. Listernick R, Mancini AJ, Charrow J. Segmental Neurofibromatosis in childhood. Am J Med Genet A 2003; 121A: 132-5.

8. Adigun CG, Stein J. Segmental neurofibromatosis. Dermatol Online J 2011; 17: 25.
9. Lara-Corrales I, Moazzami M, García-Romero MT et al. Mosaic neurofibromatosis type 1 in Children: A Single-Institution Experience. J Cutan Med Surg 2017; 21: 379-82.

10. Garcia-Romero MT, Parkin P, Lara-Corrales I. Mosaic neurofibromatosis type 1: a systematic review. Pediatr Dermatol 2016; 33: 9-17.

11. Dang JD, Cohen PR. Segmental Neurofibromatosis and Malignancy. Skinmed 2010; 8: 156-9.

12. Dang JD, Cohen PR. Segmental neurofibromatosis of the distal arm in a man who developed Hodgkin Lymphoma. Int J Dermatol 2009; 48: 1105-9.

13. Ferrer-Bruker S, Steinmetz C, Schillinger B, Usmani A. Segmental neurofibromatosis: a report of two cases and review of a rare and inconspicuous subset of a common genodermatosis. J Am Osteopath Coll Dermatol 2014; 30: 40-3.

14. Yalçin B, Toy GG, Tamer E et al. Increased expression of segmental neurofibromatosis with bronchoalveolar lung carcinoma. Dermatol 2004; 209: 342 . 